\title{
Wrapper Approach to Select a Subset of Color Components for Image Segmentation with Photometric Variations
}

\author{
Lúcio André de Castro Jorge \\ Embrapa Instrumentação Agropecuária \\ Rua 15 de Novembro, 1452, C.P. 741, 13560-970, São Carlos, SP - Brazil \\ lucio@cnpdia.embrapa.br \\ Henrique de Souza Ruiz \\ Engenharia Elétrica, Universidade de São Paulo \\ Departamento de Engenharia Elétrica, EESC-USP \\ Av Trabalhador São-carlense, 400 - 13566-590 - São Carlos, SP, Brazil \\ henriquesruiz@superig.com.br \\ Ednaldo José Ferreira \\ Embrapa Instrumentação Agropecuária \\ Rua 15 de Novembro, 1452, C.P. 741, 13560-970, São Carlos, SP - Brazil \\ ednaldo@cnpdia.embrapa.br \\ Adilson Gonzaga \\ Departamento de Engenharia Elétrica, EESC-USP \\ Av Trabalhador São-carlense, 400 - 13566-590 - São Carlos, SP, Brazil
}

\begin{abstract}
The choice of a color model is of great importance for many computer vision algorithms. However, there are many color models available; the inherent difficulty is how to automatically select a single color model or, alternatively, a subset of features from several color models producing the best result for a particular task. To achieve proper colors components selection, in this paper, it was proposed the use of wrapper method, a data mining approach, to obtain repeatability and distinctiveness in segmentation process. The result was compared with neural network method and yields good feature discrimination. The method was verified experimentally with 108 images from Amsterdam Library of Objects Images (ALOI) and 10 aerial images with different photometric conditions. Furthermore, it has shown that the color model selection scheme provides a proper balance between color invariance (repeatability) and discriminative power (distinctiveness).
\end{abstract}

\section{Introduction}

The choice of color systems is of great importance for the purpose of some computer vision algorithms. No color space can be considered as universal because color can be interpreted and modeled in different ways. It is possible that several color spaces are equally good candidates or that different color channels have similar properties (for instance, both V and $\mathrm{G}$ channels encode the intensity information for green colors). In these cases, the subsequent question is how to combine color spaces or color channels [10].

Color systems have been developed for different purposes, such as, display process (RGB), color uncorrelation $\left(\mathrm{I}_{1} \mathrm{I}_{2} \mathrm{I}_{3}\right)$, perceptual uniformity $\left(\mathrm{L}^{*} \mathrm{a} \mathrm{b}^{*}\right)$, intuitive description (HSV) and others. With this large variety of color systems, the inevitable question arises which color system to use for which kind of image application. To this end, criteria are required to classify the various color systems for the purpose of computer vision applications. Firstly, an important criterion is that the color system is independent of the underlying imaging device. This is required when images are 
recorded by different imaging devices such as cameras. Another requirement is that the color system should exhibit perceptual uniformity meaning that numerical distances within the color space can be related to human perceptual differences. This is important when images are retrieved from databases which should be visually similar. Also, the transformation needed to compute the color system should be linear, since nonlinear transformation may introduce instabilities with respect to noise causing poor accuracy when comparing to each other. Moreover, the color system should be composed of color models which are understandable and intuitive to the user. Moreover, to achieve robust and discriminative image patterns color invariance is an important criterion. Two recordings made of the same object from different viewpoints will yield different shadowing, shading and highlighting color will drastically change the photometric content of images even when they are taken from the same object. Hence, a proper computer vision application should be robust to imaging conditions discounting the disturbing influences caused by different viewpoints, object poses or illumination. In Table 1 it is shown a summary of color systems taxonomy typically used to select the proper color system for specific applications [1].

Table 1: Overview of the dependencies differentiated for various color systems. + denotes satisfied conditions; - denotes unsatisfied conditions.

\begin{tabular}{|l|c|c|c|c|c|c|}
\hline & $R G B$ & $L^{*} a^{*} b^{*}$ & $I_{l} I_{2} I_{3}$ & $H$ & $S$ & $V$ \\
\hline $\begin{array}{l}\text { Device } \\
\text { Independent }\end{array}$ & - & + & - & - & - & - \\
\hline $\begin{array}{l}\text { Perception } \\
\text { Uniform }\end{array}$ & - & + & - & - & - & - \\
\hline Linear & + & - & + & - & - & + \\
\hline Intuitive & - & - & - & + & + & + \\
\hline View Point & - & - & - & + & + & - \\
\hline Object Shape & - & - & - & + & + & - \\
\hline Highlights & - & - & - & + & - & - \\
\hline $\begin{array}{l}\text { Ilum. } \\
\text { Intensity }\end{array}$ & - & - & - & + & + & - \\
\hline $\begin{array}{l}\text { Ilum } \\
\text { SPD }\end{array}$ & - & - & - & - & - & - \\
\hline
\end{tabular}

* spectral power distribution (SPD)

In this paper, the aim was selecting a subset of color components using data mining approach. Feature selection has been used in computer vision where one or more visual features are chosen from a given initial set of candidates.

Based on the notion of class separability, several methods have been proposed to select the feature subset [8]. Therefore, in this paper, to achieve proper color component selection, it was introduced the wrapper method described by [5], [6] e [7] using training samples.

Further, two criteria are used to assess the performance of the color feature detectors: 1) (repeatability) they should be invariant (stable) under varying viewing conditions, such as illumination, shading, highlights, and 2) (distinctiveness) they should have high discriminative power. It has been shown that there exists a trade-off between color invariant models and their discriminative power [9], [10]. For a particular computer vision task that assumes only a few different light sources, color models should be selected, which are invariant (only) to these few light sources resulting in an augmentation of the discriminative power of the algorithm.

Therefore, the aim is to automatically select color models to arrive at a proper balance between color invariance (repeatability) and discriminative power (distinctiveness).

The paper is organized as follows: in Section 2, sample images sets are presented. In Section 3, it is described the wrapper method and Weka workbench. In Section 4, the experiments are described and the results and discussions are presented in Section 5. Finally, the conclusions are given in Section 6.

\section{Sample Image Set}

The obtained sample sets are referred to set I and set II:

Set I: 108 color images with $192 \times 144$ pixels of object 25 from Amsterdam Library of Objects Images (ALOI) [2]. The conditions of changing viewpoint, object pose and illumination were considered. Four images can be observed in Figure 1 .
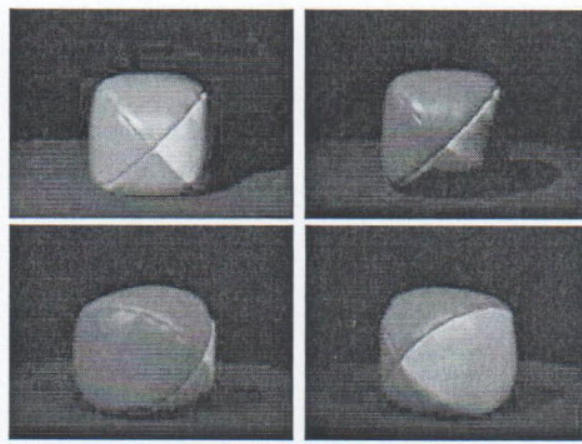

Figure 1: Object 25 from ALOI, changing viewpoint, object pose, and illumination

- Set II: 10 color aerial images with $372 \times 248$ pixels acquired from different photometric conditions. Four images from a series taken from real-world scenes of citrus farms at 100 meters high 
and at different conditions of illumination and viewpoint, are presented in Figure 2.

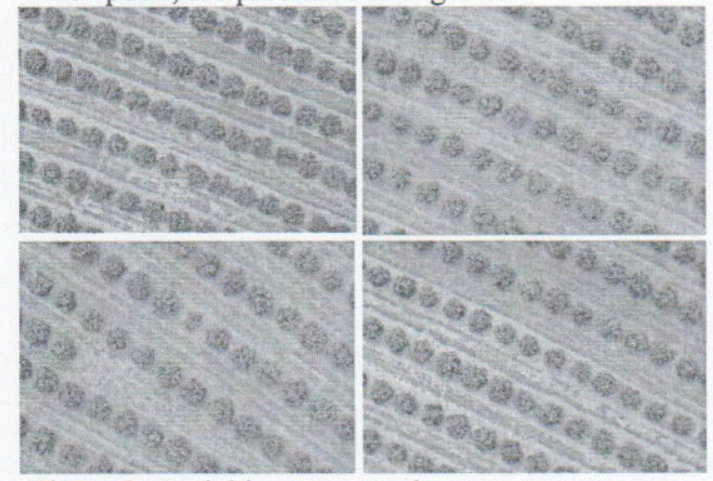

Figure 2: Aerial images samples

\section{Wrapper Method and Weka System}

In supervised learning, feature selection is often viewed as a search problem in a space of feature subsets. To carry out this search it is necessary to specify a starting point, a strategy to cross the space of subsets, an evaluation function and a stopping criterion. Although this formulation allows a variety of solutions to be developed, usually two families of methods are considered. On one hand, filter methods use an evaluation function that relies solely on properties of the data, thus is independent on any particular learning algorithm. On the other hand, wrapper methods use the inductive algorithm to estimate the value of a given subset [6]. An induction algorithm is typically presented with a set of training instances, where each instance is described by a vector of features or attributes values and a class label. The task of the induction algorithm (inducer) is to induce from training data a classifier that will be useful in classifying future cases. The classifier is a mapping from the space of feature values to the set of class values. In the feature subset selection problems, a learning algorithm is faced with the problem of selecting some subset of features upon which to focus its attention, while ignoring the rest. The idea behind the wrapper approach [5], shown in Figure 3, is simple: the induction algorithm is used as a black box. For each selected feature subset during the search process, one classifier is created by the learning algorithm. Typically, the accuracy of this classifier is used evaluate the feature subset efficiency. Therefore, the selected subset is relevant to the learning task and the algorithm [6].

Practical machine learning algorithms i.e. decision tree algorithms such as C4.5 [5], [4] and instance based algorithms such as IBL [5] have shown lower classification performance when induced from sets with a lot of irrelevant features. Thus, the feature subset selection can improve the accuracy of classifiers induced by the same algorithm used in wrapper method.

In practical learning scenarios, however, it is faced with two problems. First, the learning algorithms are usually given a relatively small training set. Second, even quite similar algorithms may incorporate different heuristics to aid in quickly building models of the training data finding the smallest model consistent

Since C4.5 is an algorithm that performs well on a variety of real databases, it is might expected to be difficult to improve upon its performance using feature selection.

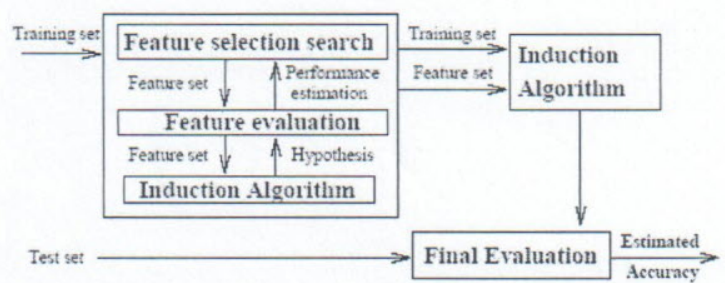

Figure 3: The wrapper approach to feature subset selection [6]

Wrapper methods are widely recognized as a superior alternative in supervised learning problems, since by employing the inductive algorithm to evaluate alternatives they have into account the particular biases of the algorithm. However, even for algorithms that exhibits a moderate complexity, the number of executions that the search process requires results in a high computational cost, especially as it is possible to shift to more exhaustive search strategies.

In this work, wrapper was implemented using Weka package. The Weka workbench is a collection of stateof-the-art machine learning algorithms and data preprocessing tools. It was developed at the University of Waikato in New Zealand. It is written in Java under terms of the GNU General Public Licence [5].

Weka attribute selection methods contain search methods such as best-first, forward selection, random, exhaustive, genetic algorithm and ranking. Contain evaluation methods such as correlation-based, wrapper, information gain, chi-squared, etc.. Classifiers in WEKA are models for predicting nominal or numeric quantities and include: decision trees and lists, instance-based classifiers, support vector machines, multi-layer perceptrons, logistic regression, Bayes' nets, etc.

In this work, it was used wrapper with an exhaustive search and embedded feature subset selection by $\mathrm{C} 4.5$ algorithm. 


\section{Experiments}

For all experiments described in this section, the RGB images were transformed into the following color channels: HSV, CIE L*a*b*, $\mathrm{I}_{1} \mathrm{I}_{2} \mathrm{I}_{3}$ [1]. All 12 color components were used in the experiments.

These models were selected as they are commonly encountered in color image processing. Further, these color models contain both variant and invariant properties with regard to the imaging conditions. RGB, CIE L*, and SV are all sensitive to shadows, shading, illumination, and highlights. Further, CIE $\mathrm{a}^{*} \mathrm{~b}^{*}$ are invariant to shadows, shading, and illumination intensity [1].

As these color channels provide both color invariance, i.e., repeatability (CIE $\mathrm{a}^{*} \mathrm{~b}^{*}$ ), and variance, i.e., distinctiveness (RGB, CIE L* and S, and V), it is allowed to test whether the proposed method will yield an optimal balance between repeatability and discriminative power by choosing the proper weights for the color channels.

A first experiment was conducted on a series of images taken from object number 25 of the Amsterdam Library of Images [2]. The image shows a ball with red, green, yellow and blue colors against a black background. Images are taken under various viewpoints and illuminations. Samples of training and test images are shown in Figure 1. The training patches were obtained from regions over each image. The defined pattern classes were red, green, yellow, and blue colors from the ball and a black background. For each pattern, five $n x n$ regions samples containing various kinds of colors: normal, very dark, and highlights were selected. In Figure 4, is shown a selection of samples for a green class over the image. This methodology was carried out for all classes and all images.

For each sample region $r$, of size $(\mathrm{I}, \mathrm{J})$ the image was decomposed in 12 images, one for each color components and the mean, variance and entropy of gray values $r(i, j)$, for $i=1 \ldots I, j=1 \ldots J$, as shown in equations (1), (2) and (3), were assessed to perform the wrapper feature subset selection. The entropy was determined by gray histogram $h(k)$ where $v(h(k))$ is gray values occurrences and $k$ is the gray level.

$$
\begin{aligned}
& \text { Mean }=\frac{1}{M N} \sum_{i=1}^{M} \sum_{j=1}^{N} r(i, j) \\
& \text { Variance }=\frac{1}{M N} \sum_{i=1}^{M} \sum_{j=1}^{N}\left(r(i, j)-\text { Mean }_{i j}\right)^{2}
\end{aligned}
$$

$$
\text { Entropy }=\sum_{i=0}^{255} h(k) * v(h(k))
$$

The feature vector $F V$ for all color components is:

$F V=[$ meanR, mean $G$, mean $B$, mean $H$, meanS, meanV, meanI $I_{1}$ meanI ${ }_{2}$, meanI $I_{3}$, mean L, meana, meanb, variance $R$, variance $G$, varianceB, varianceH, varianceS, varianceV, variance $I_{1}$, variance $I_{2}$, variance $I_{3}$, variance $L$, variancea, varianceb, entropyR, entropy $G$, entropyB, entropyH, entropyS, entropyV, entropy $I_{1}$, entropy $I_{2}$, entropy $I_{3}$, entropy $L$, entropya, entropyb]

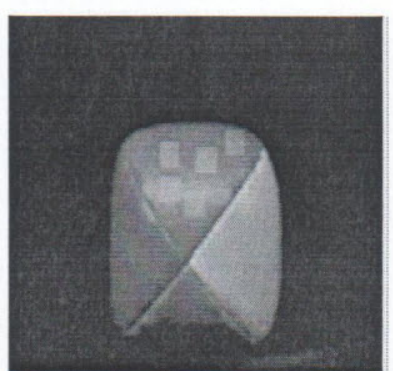

Figure 4: Selection of five $3 \times 3$ regions samples for a green class

After creating a database with all $F V$, the wrapper method was applied to select a feature subset using an exhaustive search. Also, the embedded selection provided $\mathrm{C} 4.5$ algorithm was applied.

To evaluate the performance of the selected subset, test images were segmented in regions corresponding to the pattern classes used. The segmentation process used a decision tree generated by Weka and the mean value, variance and entropy for each pixel were calculated using a kernel $3 \times 3$ over the image.

The second experiment was conducted on aerial images taken from citrus crop areas in order to identify agricultural management quality. Image segmentation can be used to identify relative differences in crop vigor, plagues, diseases and plant development level. The goal is testing a color subset selection applied to image segmentation taken at real field conditions. This kind of application is especially interesting, because it is not possible to control the sun light, shadows and highlights. The photometric conditions are not controlled.

Like the first experiment, region samples pattern were taken, but using different size selections, as 
shown in figure 5. The proposed pattern classes were citrus tree, uncover soil and weed infestation.

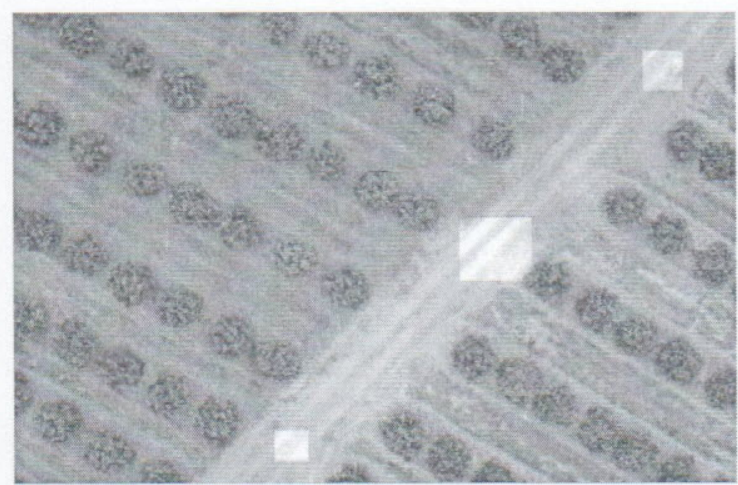

Figure 5: Selection of different size square regions samples for citrus tree, uncover soil, weed infestation classes

The training samples were repeated for all images. For each sample region $r$, the image was decomposed in 12 images, one for each color components and the mean, variance and entropy were assessed.

After $F V$ determined, it was applied wrapper to select a feature subset using an exhaustive search. The embedded selection provided by $\mathrm{C} 4.5$ algorithm also was considered.

To evaluate the performance of the selected subset, test images were segmented in regions corresponding to the pattern classes used. The segmentation process used a decision tree generated by Weka and the mean value, variance and entropy for each pixel were calculated using a kernel $3 \times 3$ over the image.

The last experiment was performed to compare wrapper with a classifier by decision tree with neural network segmentation. The same training samples from first and second experiments were applied to training a MLP neural network trained by Backpropagation algorithm, but using $F V$ reduced to the mean values only.

After training the neural network, the segmentation was performed just presenting pixel by pixel to the MLP neural network. The mean value for each pixel was calculated from a kernel $3 \times 3$ over the image.

\section{Results and Discussions}

The proposed method has been tested on a wide variety of conditions. First, the proposed selection of color components using wrapper with an embedded C4.5 algorithm was applied to the ball with different photometric conditions. The first two ball images have different color temperature; the third highlights from illumination and the last one, rotation from viewpoint, as shown in Figure 6. The exhaustive search has algorithm complexity $\mathrm{O}\left(2^{\mathrm{n}}\right)$, where $\mathrm{n}$ is the number of features corresponding to 12 means, 12 variances and 12 entropies. That is why only the means were preselected. The accuracy using 10-fold cross-validation was $98.98 \%$ and selected features were $\mathrm{I}_{2}, \mathrm{a}, \mathrm{I}_{3}$ and $\mathrm{G}$ means. The results of segmentation with the decision tree generated are shown in Figure 6, and the decision tree is shown in Figure 7.

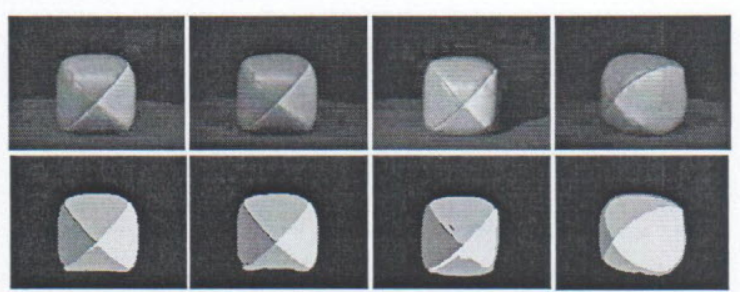

Figure 6: Original images of ALOI object 25 and the results of image segmentation based on wrapper with $\mathrm{C}$ 4.5 decision tree algorithm.

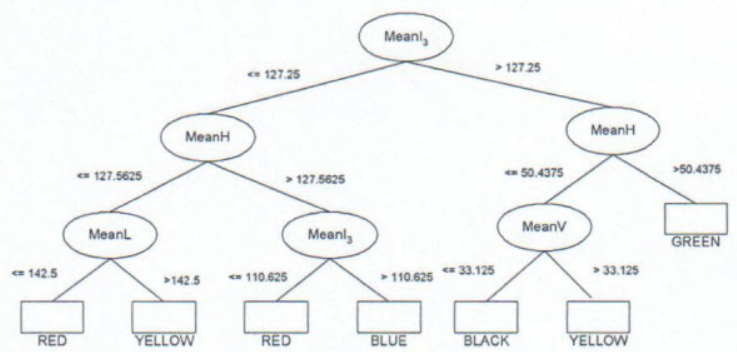

Figure 7: Decision tree for ALOI object 25

Another test using a new training set (new samples) and the wrapper approach (exhaustive search) with C 4.5 was tried to include variances and entropies. The experiment was divided in two steps. First, an exhaustive search only with the means was applied. In this case, the feature subset selection selected $\mathrm{I}_{3}, \mathrm{H}, \mathrm{L}$ and $\mathrm{V}$. The second step adds the respective variance and entropy up i.e. those corresponding to $\mathrm{I}_{3}, \mathrm{H}, \mathrm{L}$ and $\mathrm{V}$. The accuracy obtained with the best feature subset was $99.62 \%$ ( 10 -fold cross-validation).

In spite of new features (variances and entropies) added up, it was obtained the same color subset of components $\mathrm{I}_{3}, \mathrm{H}, \mathrm{L}$ and $\mathrm{V}$, and consequently the same decision tree. This indicated that, for this kind of images, variance and entropy don't contribute for classification very much. The segmentation results are shown in Figure 8. 


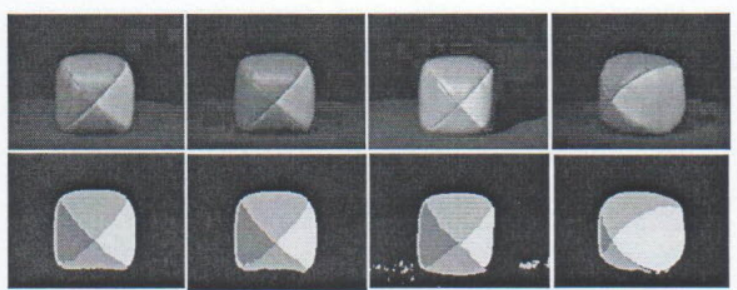

Figure 8: Original images of ALOI 25 and the results of image segmentation based wrapper at exhaustive search method

Using the same decision tree, it was tested others images from ALOI and football soccer databases that have the same colors but that had not used to generate the decision tree. The algorithm performance can be observed in Figure 9.

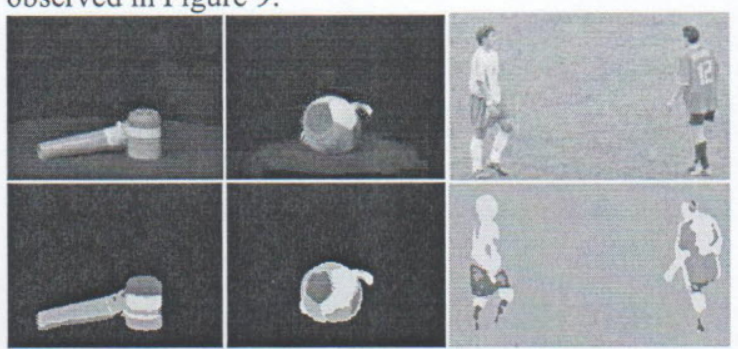

Figure 9: The same decision tree considered the best for this application and showed in Figure 6.

The second experiment has applied to aerial images. The proposed selection of color components using the wrapper approach with an embedded $\mathrm{C} 4.5$ algorithm was used for all color components. In this case, the feature subset selection algorithm achieves accuracy of $99.52 \%$ and the selected features were mean $\mathrm{G}$, mean a and entropy B. This decision tree was obtained using two step procedure, like before. Nevertheless, all entropies and variances were added to the means. The results of segmentation with the decision tree (Figure 10) are shown in Figures 11.

Finally, the wrapper approach with exhaustive search was applied to all features at once. The exhaustive search in Weka follows an organized search strategy which selects a new feature subset just if the current subset selected is exclusively worse than the new candidate. Thus, the selected features were $\mathrm{a}^{*}$ and $\mathrm{L}$ with the same accuracy obtained before $(99.52 \%)$. However, the results have shown a worse performance for segmentation process (Figure 13).

The wrapper has the potential to make an accurate selection but experiments suggest evidence that it is too prone to get trapped in local maxima, a well known problem for forward search strategies. The solution for this is the exhaustive search.

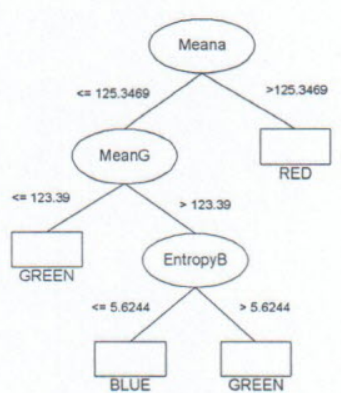

Figure 10: Decicion tree for aerial citrus images

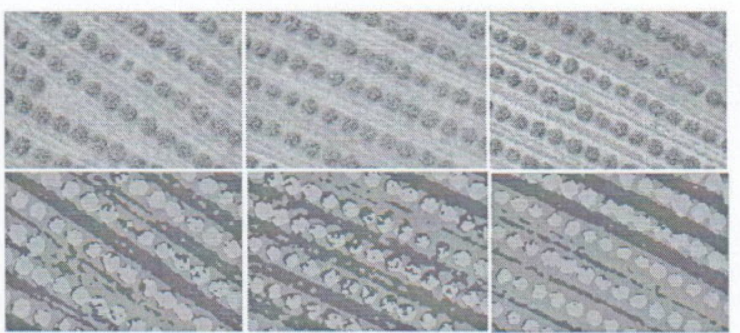

Figure 11: Aerial citrus images and the result using the decision tree from Figure 10.

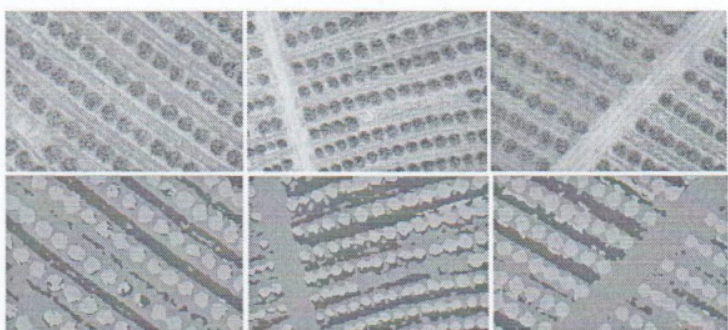

Figure 12: Aerial citrus images and the result using the decision tree from Figure 10.
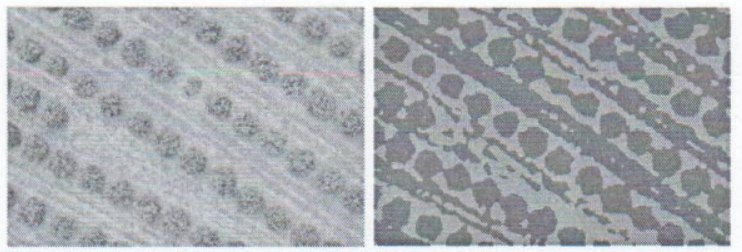

Figure 13: Segmentation of aerial citrus image for wrapper at exhaustive search method.

The main task of wrapper is to find the shortest and accurate subset selection. The results suggest that with embedded $\mathrm{C} 4.5$, is possible to have more solutions.

The best solution is that found first.

The results from those experiments were observed by visual inspection. For balls images, it was easy to identity if it was good or not instead of for aerial images. 
In order to explore more details about Wrapper and compare with other methods, it was implemented a MLP neural network as a segmentation algorithm. The results for balls and aerial images could be observed in Figures 14,15 e 16 . The neural network was trained with the same mean RGB vector and presented a training error less than $0.1 \%$. It is possible to observe that the results show more problems to uncover soil and weeds. For balls, photometric conditions could not be a problem.

The main goal here went to evaluate a segmentation system with the RGB components once the other color systems are derived from this and they present some redundancy. An appropriate acting was observed in the segmentation of the ALOI 25 object, showing that even under different illumination conditions, color temperature and point of view, it is possible to segment them with little color components. However, in more complex images, as the aerial images, zeroth order statistics can be an important and necessary factor, besides the invariant components. Furthermore, the selection of the colors components provided by the Wrapper approach stands out, presenting better acting even with simple algorithms of classification (C4.5) compared to the traditional models of neural nets.

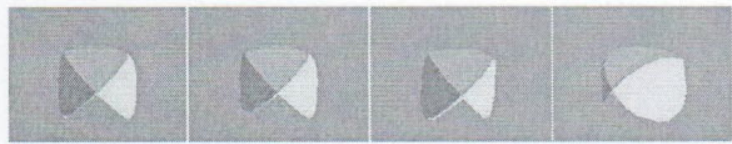

Figure 14: Segmentation of balls images using the MLP Neural Network with mean values of RGB.

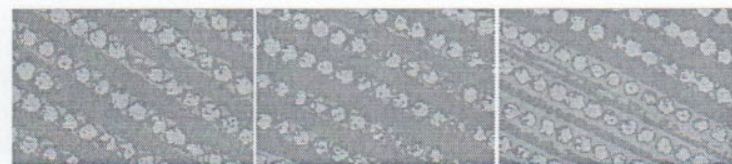

Figure 15: Segmentation of aerial citrus images using the MLP Neural Network with mean values of RGB.

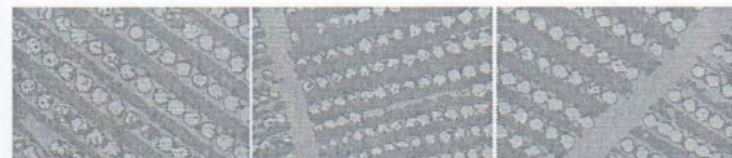

Figure 16: Segmentation of aerial citrus images using the MLP Neural Network with mean values of RGB

Another test changing wrapper with different search strategy was implemented, the instance based algorithm [5], with Euclidean distance and the results get better, as presented in Figures 17 and 18.

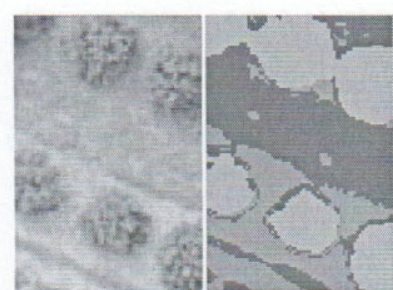

Figure 17: Segmentation of aerial citrus images for wrapper with instance based method of search, with Euclidean distance.

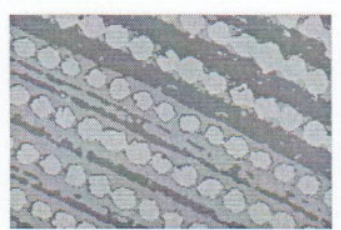

Figure 18: Segmentation of the third aerial citrus image of Figure 11, for wrapper with instance based method of search.

There exists redundancy among some color components of the different color models involved in this work, although the combination of these can improve the precision of the algorithms of machine learning. However, this work shows that the appropriate selection of a subset of color components contributes to the balance between the repeatability and distinctiveness, what is desirable in the segmentation of aerial images.

The correlation between the different color channels reveal that taking combinations of color channels performs better than taking only a single color space.

\section{Conclusions}

In this paper, a wrapper method was proposed to select a subset color models for discriminatory and robust image segmentation. Further, the method was experimentally verified that the selection model can be applied successfully to select compared with other methods. The subset selection permits proper balance between color invariance (repeatability) and discriminative power (distinctiveness).

Recently there has been a growing interest in feature selection for clustering, a number of questions still remain open. Wrappers for feature selection have been recently proposed with some success. This work improves a little with more examples. Many examples of these approaches are focused on numerical clustering, and there is no theoretical or experimental evidence related to their behavior on color images data.

The extensive experiments conducted on a wide variety of images show that proposed method is widely applicable.

\section{References}


[1] Gevers, T. Color in image search engines. University of Amsterdan. Principles of Visual Information Retrieval, Spring-Verlag, London. 2001

[2] http://staff.science.uva.nl/ aloi/

[3] http://www.cs.waikato.ac.nz/ml/weka

[4] Quinlan, J. R. (1993). C4.5: Programs for Machine Learning. Morgan Kaufmann. San Francisco, CA

[5] Witten, I.H., Frank, E. Data Mining - Practical Machine Learning Tools and Techniques with Java. Morgan Kaufmann Publishes, ( WEKA)

[6] Kohavi, R, John, G H. (1997) Wrappers for feature subset selection. Artificial Intelligence. 97(12):273-324.

[7] Kohavi, R., Sommerfield, D. (1995) Feature subset selection using the wrapper model. Overfitting and dynamic search space topology. In: The First International Conference on Knowledge Discovery and Data Mining. Pages 192-197.

[8] Duda, R.O., Hart, P.E., Stork, D.G. Pattern Classification. Willey Interscience, USA $2^{\text {nd }}$ ed. 2001

[9] Gevers, T., Stokman, H. M. G. Classification of color edges in video into shadow-geometry, highlight, or material transitions, IEEE Trans.Multimedia, vol. 5, no. 2, pp. 237-243, Jun. 2003.
[10] Stokman, H and Givers, T. Selection and fusion of color models for image feature detection. IEEE Trans. On Pattern analysis and Machine Intelligence. Vol. 29, n.3, march 2007. 